\title{
Survivin Gene Functions and Relationships between Expression and Prognosis in Patients with Nasopharyngeal Carcinoma
}

\author{
Jun-Hong Cai ${ }^{1,2}$, Sheng-Miao Fu ${ }^{2 *}$, Zhi-Hua Tu², Li-Qun Deng ${ }^{2}$, Zhu Liang ${ }^{2}$, \\ Xin-Ping Chen ${ }^{2}$, Xuan-Ju Gong ${ }^{2}$, Li-Hua Wan ${ }^{1 *}$
}

\begin{abstract}
This study aimed to investigate the relationship between prognosis and protein and mRNA expression of an apoptotic inhibitor gene, survivin, in patients with nasopharyngeal carcinoma. Furthermore, functions of the survivin gene in the CNE2 nasopharyngeal carcinoma cell line were assessed. Immunohistochemistry and in situ hybridization were used in detecting the survivin protein and mRNA in 44 nasopharyngeal carcinoma specimens, and 30 chronic nasopharyngitis samples as controls. Survivin gene expression in CNE2 cell line was suppressed with an shRNA (short hairpin RNA). The positive ratios of expression for survivin protein and mRNA in nasopharyngeal carcinoma were $\mathbf{7 9 . 5 \%}$ and $\mathbf{7 5 . 0 \%}$ respectively, obviously higher than in the control group $(p<0.01)$, and there is very good consistency between the two methods. The mean survival time of patients with higher survivin protein or mRNA expression was shorter than in patients with lower levelsv(p<0.01). Proliferation of the CNE2 cell line was distinctly inhibited by the shRNA. The results indicate that overexpression of the survivin gene plays an important role in onset and development of nasopharyngeal carcinoma, and it may be helpful for prognostic appraisal.
\end{abstract}

Keywords: Survivin gene - nasopharyngeal carcinoma - prognosis

Asian Pac J Cancer Prev, 16 (6), 2341-2345

\section{Introduction}

Nasopharyngeal carcinoma (NPC) is a frequent malignant tumor in South China and Southeast Asia. Not clarifying the nosogenesis up to now, the therapeutic effect of NPC is not very good and five year survival rates of the patients fluctuates from $50 \%$ to $60 \%$ yet. The survivin gene was found as an apoptotic inhibitor gene in 1997 (Ambrosini et al., 1997), locating on 17p25 of human chromosome. It is one member of the inhibitor of apoptosis (IAPs), can directly combine with Caspase-3 and Caspase-7 et al through the Baculoviral IAP repeat (BIR), participating the core mechanism of cell apoptosis and inhibiting it.

In this study we purpose to explore the relationship between survivin gene and prognosis of NPC patients by detecting the expression products of apoptotic inhibitor gene, survivin protein and mRNA in biopsy tissues of nasopharyngeal carcinoma, and further the functions of survivin gene in nasopharyngeal carcinoma cell line CNE2.

\section{Materials and Methods}

\section{General information}

44 cases were come from the patients consulting in the Otolaryngological department, Hainan Provincial People's Hospital from May, 2006 to September, 2007. Their diagnoses were identified by pathology with the biopsy tissue of their pars nasalis pharyngis. Among them, 37 cases' diagnoses were poor differentiated squamous cell carcinoma, 7 cases' differentiated non keratinizing carcinoma, 30 cases were male, 14 female, and their mean age were 48 (from 22 to 77 ). Their clinical stages according to Fuzhou Stages '92, 11 cases were stage II, 18 cases were stage III and 15 cases were stage IV. All patients were hospitalized for radiotherapy after conformed diagnosing, and follow-up surveyed till October, 2010. 30 cases of chronic nasopharyngitis in corresponding period were treated as the control group.

\section{Reagents}

Poly-L-lysine slide, Rabbit anti-Human 
Survivin Polyclonal Antibody, UltraSensitive S-P immunohistochemical staining kit, diaminobenzidine (DAB) chromogenic reagent kit were purchased from Fuzhou Maixin Biotechnology Development Limited Company. Survivin mRNA in situ hybridization kit was purchased from Wuhan Boster Bio-engineering Limited Company. Nasopharyngeal carcinoma cell line CNE2 was purchased from China Center for Type Culture Collection. The specifical oligo DNA (shRNA), which could interfere the survivin gene, was designed and synthesized by Wuhan Genesil Biotechnology Limited Company.

\section{Specimen storage and preparation}

The biopsy tissues were embedded by paraffin after being fixed by $10 \%$ formalin. They were sectioned by the microtome at $5 \mu \mathrm{m}$ per slice.

\section{Survivin protein detected by immunohistochemistry}

It was detected by SP method. Following dewaxing, hydrating, and repaired by citric acid tissue antigen repairing liquid, incubated in endogenous peroxidase blocking solution at room temperature for 10 minutes. Then, after incubating in normal nonimmune animal serum at room temperature for 10 minutes, combined with rabbit anti-human survivin polyclonal antibody at $4^{\circ} \mathrm{C}$ overnight. Incubated in the streptavidin-biotin-peroxidase solution at room temperature for 10 minutes, stained by DAB, counterstained with hematoxylin, dehydrating and transparentizing, observed by microscope after mounting.

\section{Survivin mRNA detected by hybridization in situ}

The process before hydrating was as same as immunohistochemistry. The tissues' endogenous peroxidase was inactivated. Digested by pepsase and fixed by $1 \%$ paraform, prehybridized at $39^{\circ} \mathrm{C}$ for two hours, hybridized at $39^{\circ} \mathrm{C}$ overnight, blocked at $37^{\circ} \mathrm{C}$ for 30 minutes, combined with biotinylated mouse antidigoxin solution at $37^{\circ} \mathrm{C}$ for one hour. After reacting with streptavidin-biotin-peroxidase complex at $37^{\circ} \mathrm{C}$ for 20 minutes, incubated in biotin peroxidase solution at room temperature for 30 minutes, stained by DAB, counterstained with hematoxylin, dehydrating and transparentizing, observed by microscope after mounting.

\section{Microscopic evaluation and findings}

Counting 100 tumor cells in each of 5 visual fields of the high powering microscope at $400 \times$. The tumor cells with yellow pellets or patches in cytoplasm or nucleus are identified as the positive. Calculating the ratio of positive cells in all nasopharyngeal carcinoma cells, if the ratio exceeded $50 \%$, the case would be marked as (+++), from $26 \%$ to $50 \%$ marked as (++), from $6 \%$ to $25 \%$ marked as $(+)$, below or equal to $5 \%$ marked as (-), among all the cases, greater than $25 \%$ identified as high expression, from $0 \%$ to $25 \%$ low expression.
shRNA interfering experiments of survivin gene

CNE2 cell line was cultured in Minimum Essential Medium (MEM) with $2 \mathrm{mM}$ L-glutamine, $1.5 \mathrm{~g} / \mathrm{L}$ sodium bicarbonate, $0.1 \mathrm{mM}$ non-essential amino acids, and $1.0 \mathrm{mM}$ sodium pyruvate, $10 \%$ fetal bovine serum. The oligo DNA (shRNA), with enzyme loci at the two terminals, was cloned into vector pU6-M4 and verified by sequencing. The vector was transfected into CNE2 by Lipofectamine 2000, and the survivin mRNA was detected by fluorescence quantitative RT-PCR after 24 hours with the primers, 5'-TCAAGGACCACCGCATCTCTA-3' and 5'-TGAAGCAGAAGAAACACTGGGC-3', $\beta$-actin gene as the control with the primers, 5 '-GTGGTGGTGA A GCTGTAGCC-3' and 5'-GAGACCTTCAACACCC-3'. The method of $2^{-\Delta \Delta C t}$ (Circle of threshold) was applied in analyzing the relative mRNA quantitation of the two genes. At the same time, the proliferation of CNE2 cells were detected by the method of MTT assay.

\section{Statistical analysis}

Four grid data was proceeded by $\chi^{2}$ test and comparison of two group data was analyzed by t test with Statistics Analysis Software (SAS) 8.0.

\section{Results}

Expression of survivin in nasopharyngeal carcinoma tissues. The expression of survivin protein was: 20 cases $(+++), 10$ cases $(++), 5$ cases $(+), 9$ cases $(-), 30$ cases of high expression, and 14 cases of low expression (Figure 1). By contrast, only 11 cases were marked as (+) among 30 cases of the control group, biopsy tissues of chronic nasopharyngitis in corresponding period. And the hybridization in situ of survivin mRNA was: 14 cases $(+++), 13$ cases $(++), 6$ cases $(+), 11$ cases $(-), 27$ cases of high expression, and 17 cases of low expression (Figure $2) .10$ cases were marked as (+) in the control group. Details were in the Table 1. Moreover, there was good coincidence between the expression level of survivin protein and mRNA, that is, the ratio of co-negative cases (9 cases) and co-positive cases ( 32 cases), which detected by the two methods, reached $90.9 \%$.

The relationship between the expression level of survivin gene and the survival time of patients with

Table 2. The Relationship between the Expression Level of Survivin Gene and the Survival Time of Patients with Nasopharyngeal Carcinoma $(\overline{\mathbf{x}} \pm \mathbf{s})$

\begin{tabular}{lccc}
\hline Testing item & $\begin{array}{c}\text { Expression } \\
\text { level }\end{array}$ & $\begin{array}{c}\text { Mean survival } \\
\text { time(month) }\end{array}$ & $p$ value \\
\hline Survivin protein & high & $28.9 \pm 11.7$ & $<0.01$ \\
& low & $44.5 \pm 3.6$ & \\
Survivin mRNA & high & $29.9 \pm 12.4$ & $<0.01$ \\
& low & $40.1 \pm 10.8$ & \\
\hline
\end{tabular}

Table 1. The Expression Ratio of Survivin Protein and mRNA (\%)

\begin{tabular}{lccr}
\hline Groups & Cases & Positive ratio of survivin protein & Positive ratio of survivin mRNA \\
\hline Nasopharyngeal carcinoma & 44 & $79.5(35 / 44)$ & $75.0(33 / 44)$ \\
chronic Nasopharyngitis & 30 & $36.7(11 / 30)$ & $33.3(10 / 30)$ \\
Statistical process & & $\chi^{2}=13.94, p<0.01$ & $\chi^{2}=12.72, p<0.01$ \\
\hline
\end{tabular}


Table 3. The Relative Quantitations of Survivin Mrna and Inhibition Rate of CNE2 Cells afterInterfering by ShRNA $(\overline{\mathbf{x}} \pm \mathbf{s}), \mathrm{n}=4)$

\begin{tabular}{|c|c|c|c|c|c|}
\hline Groups & $\begin{array}{c}\text { relative quantitations of } \\
\text { survivin mRNA }\end{array}$ & $p$ value & OD & inhibition rate & $p$ value \\
\hline Blank group & $1.04 \pm 0.23$ & & $0.461 \pm 0.004$ & & \\
\hline Lipofectamine 2000 group & $0.98 \pm 0.13$ & $>0.05$ & $0.460 \pm 0.003$ & $0.20 \%$ & $>0.05$ \\
\hline shRNA interfering group & $0.49 \pm 0.19$ & $<0.05$ & $0.343 \pm 0.003$ & $25.60 \%$ & $<0.05$ \\
\hline
\end{tabular}

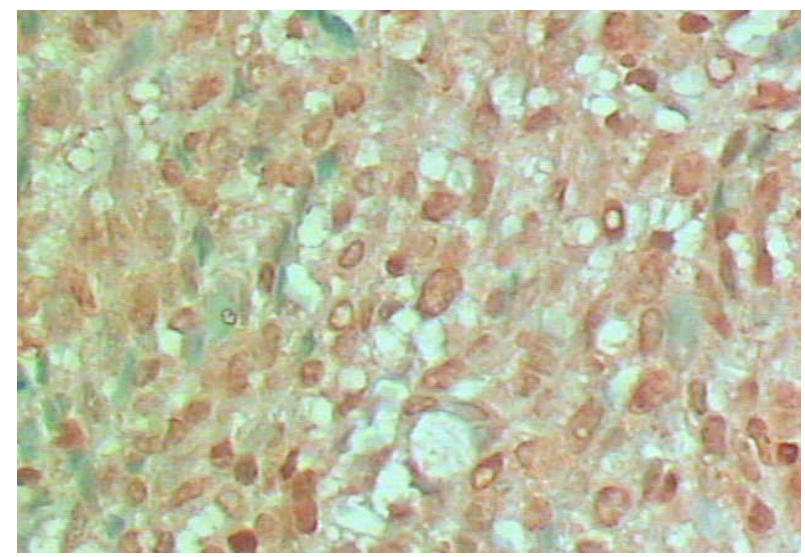

Figure 1. Positive Section of Survivin Protein in NPC Tissue (400x)

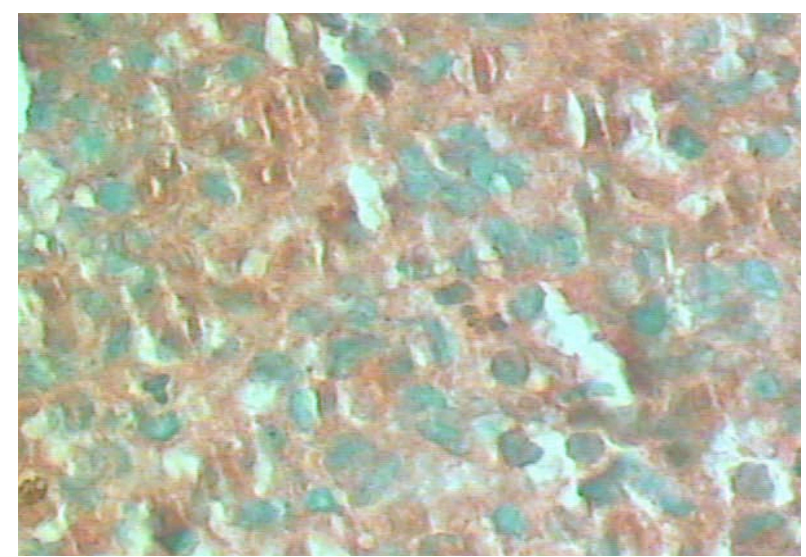

Figure 2. Positive Section of Survivin mRNA in NPC Tissue (400x)

nasopharyngeal carcinoma. The mean survival time of the patients whose survivin protein were high expression was 28.9 months, obviously shorter than 44.5 months, that of the patients whose survivin protein were low expression. Analogously, the mean survival time of the patients whose survivin mRNA were high level was 29.9 months, noticeably shorter than 40.1 months, that of the patients whose survivin mRNA were low level. Details were in the Table 2.

The sequence of the oligo DNA (shRNA) was seen in Figure 3. The results of fluorescence quantitative RT-PCR of survivin and $\beta$-actin gene show that their amplification efficiencies were consistent and dissolving curves of products were single peak. The relative quantitations of survivin in the groups of blank and Lipofectamine 2000 were not significant difference, and that of the interfering group was down-regulated about $52.9 \%$ with statistical significance $(p<0.05)$. And the proliferation activity of the interfering group was descended obviously too $(p<0.05)$. Details were in the Table 3.

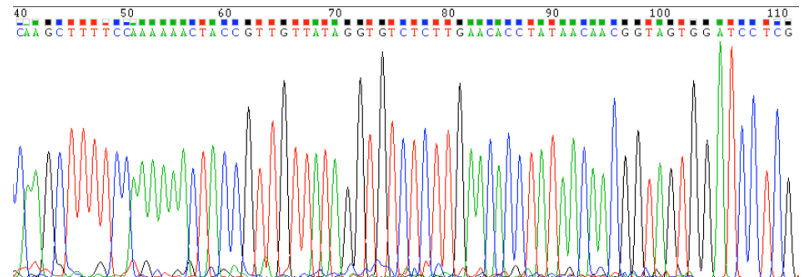

Figure 3. The Sequence of the Oligo DNA (shRNA).

41-45: Hind III restriction enzyme cutting site; 51-55: terminator; 56-75: antisense sequences; 76-82: loop; 83-102: sense sequences; 104-108: BamHI restriction enzyme cutting site

\section{Discussion}

Cell apoptosis occurs in physiological or pathological conditions. There is an intracellular inducing process, stimulated by the prompting apoptosis signal, and the mitochondrion can release cytochrome $\mathrm{C}$, which will join and activate the apoptotic protease activating factor, then the latter initiates apoptosis effect factor Caspase-9, Caspase- 3 and Caspase-7, thus gives rise to apoptosis (Cryns et al., 1999). In this process, survivin can inhibit the core mechanism involving effect factor Caspase- 3 and Caspase-7 directly to prevent from cell apoptosis (Deveraux et al., 1999). Additionally, survivin can also obstruct the mitochondrion releasing cytochrome $\mathrm{C}$, and connect with Second Mitochondria-Derived Activation of Caspase (SMAC), then protect IAPs without being inhibited by SMAC, thereby play a role in inhibiting Caspase indirectly. Survivin generally expresses in embryonic developing tissues of human or mammal and the most tumor tissues of human, but doesn't express in the normal adult tissues of differentiating maturity (Adida et al.,1998; Lu et al., 1998; Tanaka et al., 2000; Dong et al., 2002). The study of Shin et al declared that survivin was the most powerful inhibitory factor of apoptosis discovered so far, and its molecular target, Caspase was the core mechanism of cell apoptosis, with activating and dissolving proteins in cascade type, and determining the variation and trend of cell apoptosis (Shin et al., 2001). Scholars in China reported that 51 cases biopsy tissue samples of nasopharyngeal carcinoma were detected by the methods of immunohistochemistry and TUNEL (TdTmediated dUTP nick end labeling) to study the relationship between survivin protein and apoptosis index, discovering that the positive ratio of survivin protein expression in nasopharyngeal carcinoma tissues was $82.4 \%$, among them $51 \%$ was high expression, the mean apoptosis index was $2.6 \%$, and the survivin protein expression was related to the apoptosis index and prognosis of the patients closely ( $p<0.05)$ (Zhang et al., 2004). It is similar to our results 
that the positive ratio of survivin protein expression in 44 cases with nasopharyngeal carcinoma is $79.5 \%$, among them $68.2 \%$ is high expression, and the positive ratio of mRNA is $75.0 \%$, among them $61.4 \%$ is high expression. Moreover, our research team detected the survivin mRNA level by the method of real time fluorescence quantitative RT-PCR (reverse transcription polymerase chain reaction), and discovered that the level of cell strain and biopsy tissues of nasopharyngeal carcinoma is higher than that of chronic nasopharyngitis tissues $(p<0.05)$. This paper was already published on other journal (Fu et al., 2007). Those results point out that the survivin, regarded as apoptosis inhibiting gene, plays an important role in regulating the apoptosis of tumor cell during the process of onset and developing of nasopharyngeal carcinoma.

Each patient of research group was followed up continuously after being discharged from hospital, and investigated their survival condition. Further study for the relationship between the survivin gene expression and the prognosis of patients with nasopharyngeal carcinoma was discussed in the probability of the survivin regarded as the index of prognosis judgement of nasopharyngeal carcinoma. The research results indicated that the mean survival time of patients with high survivin protein expression was 28.9 months, obviously shorter than 44.5 months, that of patients with low expression, and the mean survival time of patients with high survivin mRNA level was 29.9 months, obviously shorter than 40.1 months, that of patients with low level. Similar results were discovered in esophageal carcinoma also that the mean survival time of patients with high survivin protein expression was 9 months, obviously shorter than 30 months, that of patients with low expression (Kato et al., 2001). But in endometrial carcinoma, scholars in Turkey found that there was no statistically significant relationship between the expression indexes of survivin and survival or the prognostic factors such as grade and stage, their tumor tissues were assessed immune-histochemically too (Aksoy et al., 2014). With regard to nasopharyngeal carcinoma, although it is relatively sensitive to radiotherapy, the 5 year survival rate of patients with it was only about $50 \% \sim 60 \%$. The positive rate of survivin expression in NPC patients with lese than 5-year survival time was higher than that in NPC patients with over 5-year survival time (Zhao et al., 2008). The reason probably was that a part of nasopharyngeal carcinoma cells had resistance to radiotherapy. Other experiments verified that the survivin was the vital reason that the pancreatic cancer cells came into being radiation resistance. And in vitro experiment, the expression level of survivin mRNA was inversely proportional to the radiation sensitivity of tumor cells, and was enhanced in sublethal doses of radiation, which illustrated that the survivin was also a significant factor of inducing radiation resistance (Asanuma et al., 2003). These research results indicated that high expression of survivin may influence the cell apoptosis in evidence, and might be the vital reason why tumor cells came into being radiation resistance and tolerance against radiotherapy at the same time.

Additionally, Other scientists in China also detected the expression situation of $\mathrm{p} 53$ protein in the biopsy tissues of 58 cases with nasopharyngeal carcinoma at stage III, and found that the positive ratio of cases with survival time under 5 years was $82.1 \%$, much longer than $31.6 \%$, that of cases with survival time above 5 years $(\mathrm{Xu}$ et al., 2001). Some research results were discovered that in 157 cases with nasopharyngeal carcinoma, the deletion ratio of the second exon in p16 gene of patients with survival time under 3 years was $69.57 \%$, obviously longer than $29.10 \%$, that of patients with survival time above 3 years $(p<0.01)$ (He et al., 2009). There was a positive correlation between survivin and bcl-2 and inverse correlation between survivin and apoptotic index. The expression of survivin was associated with radiosensitivity and prognosis (Hu et al., 2008). The patients with survivin and VEGF over-expression presented lower 5-year survival rate, as compared to those of low-expression, especially in advanced stage patients. Multivariate analysis indicated that both survivin and VEGF overexpression in NPC tumor tissues were strong independent factors of poor prognosis in NPC patients ( $\mathrm{Li}$ et al., 2008). And 13 gene and 18 SNP loci were detected at the pathway about radiotherapy or chemotherapy or cis-platinum or fluorouracil of biopsy tissues in 203 nasopharyngeal carcinoma patients of initial treatment at terminal stage by the technique of polymerase chain reaction-ligase detection reaction (PCR-LDR), after survival analysis by the method of Kaplan-Meier and multifactor analysis by Cox regression, then discovered that there were 6 genotypes of independent prognosis risk factors, that is rs25487 AA, rs1136410 CC+TT、rs2032582 AT, rs2010963 CC、rs3212986 GG、rs2279744 GG+GT $(p<0.05)$ (Qi et al., 2010). Support vector machine (SVM)-based methods were used to develop a prognostic classifier for NPC and the latter was performed in an independent cohort of 1059 patients and integrated sex and expression of seven genes, including Epstein-Barr virus latency membrane protein 1 (EBV-LMP1), CD147, caveolin-1, phospho-P70S6 kinase, matrix metalloproteinase 11 (MMP11), survivin, and secreted protein acidic and rich in cysteine (SPARC) as critical factors. In multivariate analysis adjusted for age, TNM stage, and histologic subtype, the NPC-SVM classifier was an independent predictor of 5-year survival rate in the evaluated patients (Wang et al., 2011). RNA interfering was also applied in Shandong, China, Livin and Survivin shRNA eukaryotic expression vectors were constructed to co-transfect into HepG2 cells, the mRNA and protein expression of Livin and Survivin genes were reduced and the proliferation of transfected cells were inhibited and induced cellular apoptosis more effectively (Xu et al., 2013). So we think that the survivin is mainly influence the downstream effective factors Caspaes-3 and Caspase-7 et al in the pathway of cell apoptosis, and probably interacted with other related genes. But what is the correlation between them and the exact pathogenesis of nasopharyngeal carcinoma need us to take more research cases and extend the follow-up time for further study.

\section{Acknowledgements}

The project is supported by the national natural science foundation of China (30460145 \& 81060223). 


\section{References}

Aksoy RT, Turan AT, Boran N, et al (2014). Lack of relation of survivin gene expression with survival and surgical prognostic factors in endometrial carcinoma patients. Asian Pac J Cancer Prev, 16, 6905-10.

Adida C, Berrebi D, Peuchmaur M, et al (1998). Anti-apoptosis gene, survivin, and prognosis of neuroblastoma [letter]. Lancet, 9106, 882-3.

Ambrosini G, Adida C, Alteri DC (1997). A novel anti-apotosis gene, Surviving, express in cancer and lymphoma. Nature $\mathrm{Med}, \mathbf{8}, 917-21$.

Asanuma K, Moriai R, Yajima T, et al (2000). Survivin as a radioresistance factor in pancreatic cancer. Japan J Cancer Res, 11, 1204-9.

Cryns V, Yuan Y (1999). Proteases to die for. Genes Dev, 11, 1551-70.

Deveraux QL, Reed JC (1999). IAP family proteins-suppressors of apoptosis. Genes Dev, 3, 239-52.

Dong Y, Sui L, Watanabe Y, et al (2002). Survivin expression in laryngeal squamous cell carcinomas and its prognostic implications. Anticancer Res, 4, 2377-83.

Fu SM, Cai JH, Tu ZH, et al (2007). Detection of survivin mRNA in nasopharyngeal carcinoma by real-time fluorescence quantitative RT-PCR. Practical Oncol J, 6, 503-6.

He X, Song D, Chen SQ, et al (2009). Correlation between the deletion of p16 gene and prognosis of nasopharyngeal carcinoma. Oncol J, 4, 281-3.

Hu GQ, Qi WW, Wang R (2008). Expression and prognostic significance of survivin and bcl-2 in nasopharyngeal carcinoma. Acta Med Univ Sci Technol Huazhong, 1, 109-11.

Kato J, Kuwabara Y, Mitani M, et al (2001). Expression of Survivin in esophageal cancer: correlation with the prognosis and response to chemotherapy. Int J Cancer, 2, 92-5.

Li YH, Hu CF, Shao Q, et al (2008). Elevated expressions of survivin and VEGF protein are strong independent predictors of survival in advanced nasopharyngeal carcinoma. $J$ Translational Med, 1, 1-11.

Lu CD, Altieri DC, Tanigawa N (1998). Expression of a novel antiapoptosis gene, survivin, correlated with tumor cell apoptosis and p53accumulation in gastric carcinomas. Cancer Res, 9, 1808-12.

Qi B, Guo X, Wen YF, et al (2010). Correlation between the gene polymorphism of pathway about radiotherapy or chemotherapy and prognosis nasopharyngeal carcinoma with terminal stage of partial region. Chin J Can Prev Treat, 10, 755-8.

Shin S, Sung BJ, Cho YS, et al (2001). An anti-apoptotic protein human survivin is a direct inhibitor of caspase-3 and caspase-7. Biochemistry, 4, 1117-23.

Tanaka K, Iwamoto S, Gon G, et al (2000). Expression of survivin and its relationship to loss of apoptosis in breast carcinomas. Clin Cancer Res, 6, 127-34.

Wang HY, Sun BY, Zhu ZH, et al (2011). Eight-signature classifier for prediction of nasopharyngeal carcinoma survival. J Clin Oncol, 24, 4516-25.

Xu S, Zhang WY (2001). Study on the correlation between the expression of protein p53 at stage III nasopharyngeal carcinoma and its biological behavior, prognosis, p53 gene mutation and the expression of LMP-1. Chin J Pathol, 6, 456-9.

Xu W, Chang H, Qin CK, et al (2013). Impact of co-transfection with livin and survivin shRNA expression vectors on biological behavior of HepG2 cells. Asian Pac J Cancer Prev, 9, 5467-72.

Zhang S, Xiao JY, Tian YQ, et al (2004). Overexpression of survivin protein in nasopharyngeal carcinoma: correlation with apoptosis index and the prognosis. Chinese $J$ Otorhinolaryngology-Skull Base Surgery, 4, 196-9.

Zhao YT, Zhou LY, He BW, et al (2008). Expression of survivin and PCNA protein in nasopharyngeal carcinoma and their clinical significance. Modern Med J China, 9, 18-20. 